\title{
Design of Document Management System Based on SD Card
}

\author{
Biqing $\mathrm{Li}^{1}$, Suping Jiang ${ }^{2}$ a , Wenya Lai ${ }^{1,}$, , ${ }^{*}$, Yangming Chen ${ }^{1}$, Liuren Wei ${ }^{1}$, \\ Huanhua Huang ${ }^{1}$, Huiliang Huang ${ }^{1}$, Shaohong Huang ${ }^{1}$, Yankui Zhao ${ }^{1}$ \\ ${ }^{1}$ School of Information and Communication Engineering, Hezhou University, Hezhou Guangxi \\ 542899, China; \\ ${ }^{2}$ Guangxi Talent International College, Qinzhou Guangxi 535000, China. \\ a229292710@qq.com, b, *janliful@163.com,
}

Keywords: Document Management System; STC12C5A60S2; file read-write.

\begin{abstract}
As a kind of storage equipment, SD card is used most widely in recent years, used in most portable devices, and the efficient file system also brought a lot of convenience for our work. Document management system based on SD card designed in this paper is to use the STC12C5A60S2 chip as the control core, mainly include key module, the SD card read-write module, LCD module and other peripheral circuits. Use the microcontroller to drive and initialize the SD card, you can operate the files of the SD card, including files read-write operation, the file name and the contents can display through the LCD1602. The design has the advantages of easy to read the file, simple operation, wide application, etc.
\end{abstract}

\section{Design Proposal}

\subsection{Functional Requirements.}

(1) File system composition: System Record Region, FAT Region, File Register Table Region, and Data Region.

(2) The SD card root folder can be read and the system is available.

\section{System Hardware Circuit}

\subsection{System Principle.}

This design mainly adopted STC12C5A60S2 microcontroller as control chip, through the drive of SD card to use the buttons and to operate the file, to read/write the SD card inside file and through the file directory showing on LCD screen.

First, it would be used to initialize the SD card by power on. After the initialization success, it could select to open file by pressing the key. The files on the SD card were accessed in clusters. When reading the files on the SD card, it first should search for the file registration table (the directory) according to the file name. According to file form the initial cluster number could be found in the directory of the file in the DATA area, which determined the location of the file. Through the corresponding command, it could be used to read/write files and the file creation process was similar to that.

\subsection{SPI Communication Mode.}

SD card had two modes of communication: SD mode and SPI mode. The interface signals they used were not the same. In this design, SPI mode was used.

SPI was a high speed, full duplex, synchronous communication bus. It only needed to use four lines (CS, DI, SCLK and DO) to achieve data transmission, which saved the pin of the chip. At the same time, it was also made more room for the design of circuit principle diagram, which made the design job easier. Because of it, now a lot of chips used this communication protocol, such as STC12C5A60S2 used in this design. 


\subsection{Microcontroller Clock Circuit.}

This design with the internal clock to drive the microcontroller, the frequency of $11.0592 \mathrm{MHz}$ crystal oscillator was connected in the pins on the X1 and X2, and two C5 and C6 external capacitance of $30 \mathrm{pF}$ together to form a parallel resonant circuit. It connected in big feedback loop, forming microcontroller clock circuit, and crystals $\mathrm{u} 6$ provided a stable clock signal to normally work of microcontroller, as shown in figure 1:

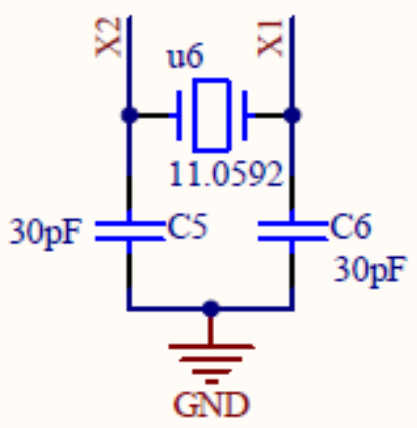

Figure 1. Clock circuit

\subsection{Reset Circuit.}

The reset circuit provided the reset functions for the STC12C5A60S2 microcontroller. When the KEY1 button was pressed, the RST foot of the microcontroller could be provided with the corresponding reset low level signal. Circuit connection was shown in figure 2:

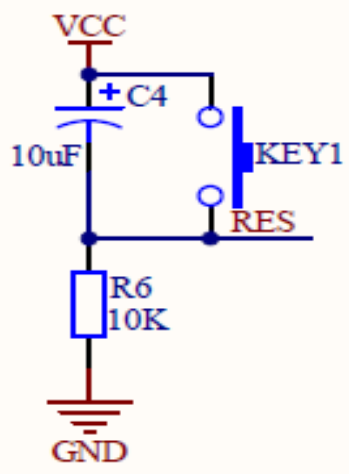

Figure 2. Reset circuit

The reset operation was essential when a microcontroller had just been on the power or when a fault occurs. The reset condition of the microcontroller: the high level with no less than two machine cycles that must be added to the RES pin. Microcontroller had two kinds of reset mode: power reset, key reset. In the power reset circuit, the reposition was realized by using capacitance charging. In power on the instant, the electric potential on the RES pin was VCC. For fast charging capacitor after power on, as the charging, RES pins on the electric potential would be gradually reduced to low level. In key reset circuit, when there was not press the button, the circuit and electric reset circuit was the same. If pressing the KEY1 in the process of single-chip running, it had charged electric capacitance could quickly through the loop discharge resistance, so that the electric potential on the RES pin quickly turned into VCC. VCC would maintain to key releases so as to meet the microcontroller reset condition to realize pushbutton reset.

\subsection{Microcontroller Minimum System.}

Microcontroller minimum system was controlled by a microcontroller, a crystal circuit, a reset circuit, and power circuit, which was a microcontroller system that could run under the minimum of peripheral circuit, as shown in figure 4. Microcontroller control circuit was the core of the whole system, to complete data processing and control tasks. 


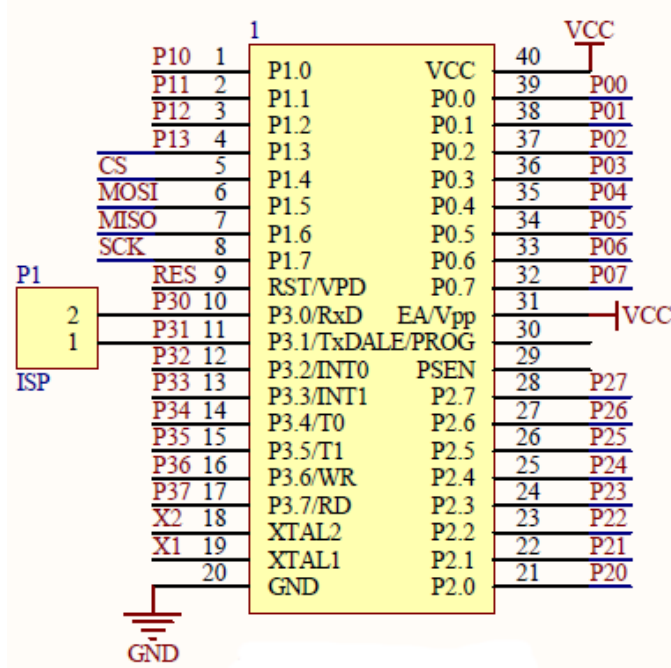

Figure 3. Microcontroller minimum system

\subsection{4-Inch TFT LCD Display Circuit.}

TFT LCD touch screen had a lot of different sizes. This design is using a 2.4 -inch LCD screen, the ILI9325 chip driver, with a touch of IC, which could realize the control by touching the screen. Compatible with $8 / 16$ bits of data interface, it could be used to display the letters, numbers, Chinese characters and images, etc., as shown in figure 4:

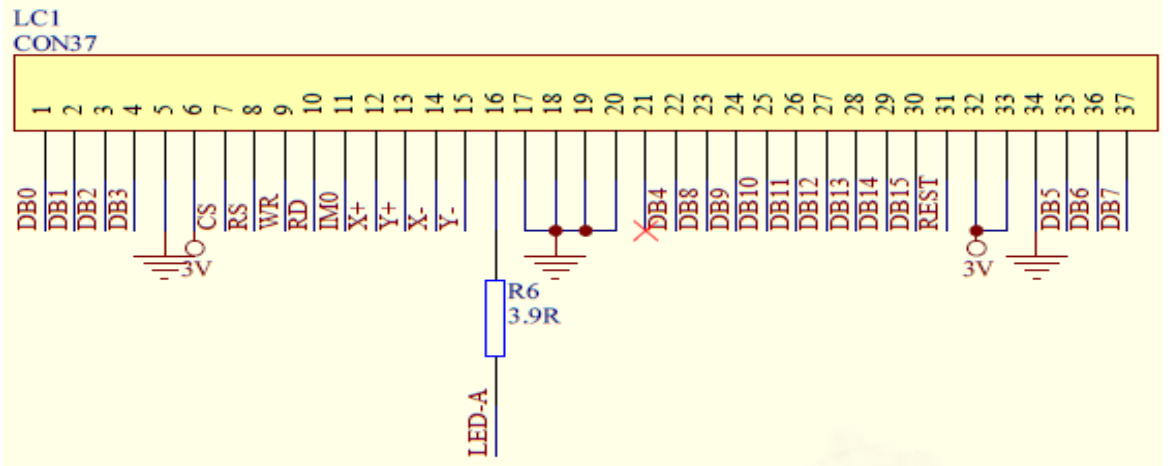

Figure 4. TFT LCD display circuit diagram

\subsection{Micro SD Card Reading and Writing Module.}

The module (Micro SD Card Adapter) was a Micro SD Card read/write module. Through the SPI interface driver, and the transplanted file system, microcontroller system could achieve read/write operation for the inside Micro SD Card files.

Module features were as follows:

(1) Supporting Micro SD card and Micro SDHC card (high speed card);

(2) Onboard level switching circuit, supply power was $4.5 \mathrm{v} \sim 5.5 \mathrm{~V}$, and onboard $3.3 \mathrm{~V}$ voltage stabilizer circuit;

(3) The communication interface was the standard SPI interface;

(4) Control interface: a total of 6 pins were configured. MISO, MOSI, and SCK were SPI data bus. GND was ground wire, VCC was supply power, and CS was selected signal pin;

(5) Level conversion circuit: The Micro SD card direction of signal was converted into $3.3 \mathrm{~V}$, and the direction of Micro SD card should follow control interface of MISO. Signal was transformed into $3.3 \mathrm{~V}$, and general 51 microcontroller system could read the signal;

(6) Micro SD clamping holder: it was self-bounce card holder, convenient for card insertion.

\section{System Software Design}

SD card driver and initialization. 
As soon as the power was on, the SD card was used by the standard SD bus protocol. When the host commands $0(\mathrm{r}=\mathrm{SD}$-Write_Cmd (pCMD0)), the SD card would detect the SPI mode to select command and switched the SD card to the SPI mode. In order to ensure that the command 0 and all other SPI mode commands were transmitted without exception, the CS pins cold not be in VCC state. When the busy status was set to VCC, the SD card was in a busy state, and the write command would fail. In order to ensure a good compatibility with SD card, the SPI clock frequency must be less than $400 \mathrm{KHz}$ when the SPI serial port and SD card were initialized.

\section{Debugging and Performance Analysis}

Connecting the power supply and turning on the switch, electricity initialized the SD card. When SD card did not insert, TFT screen was shown in figure 11. This design on the SD card was stored in the root directory of a $262 \mathrm{~KB}$ font file, so it could display Chinese characters, characters, etc. After the program was successful, this design had achieved the requirements of graduation design. Namely, the system realized the scan and display of the root directory and could read SD files normally to reach the expected aim.

\section{Conclusion}

In this design, it reviewed and consolidated the previous knowledge to learn the new while reviewing the old.

\section{Acknowledgments}

This work is supported the following fund:

2017 The student work subject program of Huizhou University: "college students' network addiction of the student work service research" (No, hzxysz201707).

2016 The project of improving the basic ability of young teachers in Colleges and universities in Guangxi:"An empirical study of Guangxi Beau Gulf Economic Zone Foreign Trade Company foreign trade English correspondence" (No, KY2016YB886).

2016 The higher education research subject project of Huizhou University: "between collaborative innovation researches of entrepreneurial talent cultivation system".

2017 college students' innovative entrepreneurial project: "The agricultural robot autonomous operations research based on machine vision"(No,201711838096) \& \&"Multi-functional autonomous agriculture robot development"(No,201711838121) \&\&"Research and development of navigation system for agricultural robot"(No,201711838008) \&\&"Research and development of data acquisition system and servo control system for mobile platform of agricultural robot" (No,201711838075) \&\&"Research and development of visual navigation system for agricultural robot test platform"(No,201711838076).

2017 Guangxi education science 'ten-three-five' planning project: "Based on set's ICT education platform of fusion, work-integrated learning education training mode research" (No, 2017B107).

\section{References}

[1]. B.Q Li, Y.F Ling, H.Y Zhang, S.Y Zheng: The Design and Realization of Cherry Tomato Harvesting Robot Based on Iota. International Journal of Online Engineering, 12(12), 23, (2016).

[2]. B.Q Li, W.L Guan, S.Y Zheng, X.G Yue: Optimisation Design of Corn Precision Seder Based on Multi-Route and Multi-Channel Control. Journal of the Balkan Tribological Association, 21(4a), 1215, (2015).

[3]. S.Y Zheng, Et Al, Social Work in Teen Addiction Correction Services Research Under the New Situation, In: Proceedings of the 2015 4th National Conference on Electrical, Electronics and Computer Engineering (Niece 2015), Xian, China, 2015, Pp.252-255. 
[4]. B.Q Li, et al, Intelligent Control Management System and Its Application, in: Proceedings of the 2016 International Conference on Economics and Management Innovations, Wuhan, China, 2016, Pp.68-71.

[5]. B.Q Li, Et Al, Design and Implementation of Tanks War Game Based on the Android Platform, In: Proceedings of the 2016 2nd Workshop on Advanced Research and Technology in Industry Applications, Dalian, China, 2016, PP.963-966.

[6]. B.Q Li, ET AL, Design OF A Tea Garden Ant freezing Control System, IN: Proceedings of the 2016 6th International Conference on Machinery, Materials, Environment, Biotechnology and Computer(Mimic), Tianjin, China, 2016, Pp.736-738.

[7]. B.Q Li, et al, Design of Electronic Compass, in: Proceedings of the 2016 6th International Conference on Machinery, Materials, Environment, Biotechnology and Computer (Mimic), Tianjin, China, 2016, Pp.1240-1243.

[8]. B.Q Li, et al, Research of Automatically Light-Adjusting Lamp, In: Proceedings of the 2016 International Conference on Computer Engineering, Information Science \& Application Technology (Icier 2016), Guilin, China, 2016, Pp.249-252.

[9]. B.Q Li, et al, The Design and Realization of Fruit Harvesting Robot Based on IOT, In: Proceedings of the 2016 International Conference on Computer Engineering, Information Science \& Application Technology (Icier 2016), Guilin, China, 2016, PP.261-264.

[10]. B.Q Li, et al, A New Type of Automatic Opening and Closing Light-Operated Curtain, In: Proceedings of the 2016 International Conference on Mechatronics Engineering and Information Technology(Iceman), Xian, China, 2016, PP.66-69.

[11]. B.Q Li, et al, Design of the Intelligent Air Humidifier, In: Proceedings Of the 2016 International Conference on Mechatronics Engineering and Information Technology (Iceman), Xian, China, 2016, PP.201-203.

[12]. B.Q Li, et al, The Design Implementation of the App of Experiencing Guangxi Folk Custom, In: Proceedings Of the 2016 International Conference on Economics and Management Innovations, Wuhan, China, 2016, PP.47-50.

[13]. S.Y Zheng, et al, Digital Display Design of Ethnic Clothing of Nanking, In: Proceedings Of the 4th International Conference on Mechatronics, Materials. Chemistry and Computer Engineering 2015(Icmmcce 2015), Xian, China, 2015, Pp.2805-2808.

[14]. S.Y Zheng, et al, Design and Implementation of Supermarket Personnel Management System Based on Java, In: Proceedings of the 2015 International Conference on Education, Management and Computing Technology, Tianjin, China, 2015, Pp.1724-1727.

[15]. S.Y Zheng, et al, Analysis of Internet of Things Talent Training and Curriculum System Innovation, In: Proceedings of the 2016 International Conference on Education, Management and Computing Technology(Icemct-16), Hangzhou, China, 2016, Pp.957-960.

[16]. S.Y Zheng, et al, Brief analysis on "HeYuanTong" Campus Mobile Phone App Design, In: Proceedings of the 2015 5th International Conference on Computer Sciences and Automation Engineering, Sonya, China, 2015, Pp.151-154. 